\title{
Global lithospheric magnetic field modelling by successive regional analysis
}

\author{
E. Thébault \\ GeoForschungsZentrum, Telegrafenberg, 14473, Potsdam, Germany
}

(Received August 10, 2005; Revised November 18, 2005; Accepted November 22, 2005; Online published April 14, 2006)

\begin{abstract}
Present lithospheric field models, like the MF4 and CM4, are produced by least squares estimation using spherical harmonic basis functions with global support. Accounting for the different properties of magnetic data at low and high latitudes, a method that can take regional differences into account is proposed. Using four years of CHAMP satellite data, a detailed lithospheric magnetic field snapshot is obtained at $400 \mathrm{~km}$ altitude over the entire sphere by stitching together a dense coverage of regional models. The individual forward models computed on a quasi-regular grid over the Earth are then transformed to spherical harmonics by direct integration. Despite the stitching procedure, the long wavelength lithospheric features are correctly reproduced and small scale features are well resolved. Without regularization, the resulting model is stable to spherical harmonic degree 56. In addition to accounting for regionally varying noise levels, the proposed technique is also well suited to deal with incomplete data coverage issues when combining satellite with near surface data. The method could therefore make an important contribution to one of the main goals of the Swarm mission: to close the spectral gap between satellite and near-surface magnetic surveys.
\end{abstract}

Key words: Regional modelling, Revised Spherical Cap Harmonic Analysis, lithospheric field, geomagnetism.

\section{Introduction}

With the advent of the satellite era, data distribution is dense and homogeneous around the Earth. Thanks to the CHAMP and the forthcoming Swarm missions (ESA report, 2004), we are now close to detect the complete long wavelength lithospheric field signal at satellite altitudes. Despite the good data quality, studying the lithospheric field signal at scales of a few tens of kilometers remains a challenging problem in geomagnetism. The model accuracy is tightly related to data precision and distribution. However, given a finite dataset, the mathematical tools used to represent the observations are equally important and schemes other than the finite spherical harmonics representation may be more appropriate to better highlight the small magnetic field wavelengths.

Geomagnetic data are generally represented by means of a finite set of spherical harmonic Gauss coefficients. Because of high order harmonics aliasing, the level at which the series is truncated affects the values of the highest degree Gauss coefficients. As a result, lithospheric models are unstable beyond degree 90 (Maus et al., 2006). The lithospheric field is comparatively weak at satellite altitudes, where other fields predominate, and models are extremely sensitive to noise. This noise may be caused, for instance, by a pure Gaussian independent errors with zero mean, biased error due to instrumentation and artifacts such as the line level problem of adjacent satellite tracks. In addition, most of the noise results from unknown and un-modelled magnetic field sources. The latter can be time-varying, spa-

Copyright (C) The Society of Geomagnetism and Earth, Planetary and Space Sciences (SGEPSS); The Seismological Society of Japan; The Volcanological Society of Japan; The Geodetic Society of Japan; The Japanese Society for Planetary Sciences; TERRAPUB. tially localized in the Polar Regions or near the equator, and are non Gaussian. Since the Gaussian assumption is violated, the usual least-squares approach no longer holds and parameter estimation can be erroneous and not robust without a priori information Menke (1989, chapter 3). A possible way to remedy this is to refine the inverse problem. For instance, data may be weighted iteratively (Sabaka et al., 2004) or other statistical methods that give less weight to outliers like the $L_{\mathbf{1}}$-norm may be used Menke (1989, chapter 3 , page 38). Unfortunately, estimating the variances and the data correlation is difficult. Besides, the $L_{1}$-norm inverse problem is not convenient to implement numerically, especially when lots of data have to be dealt with, and the maximum likelihood solution is non-unique. Furthermore, even given an ideal situation where consistent, sufficient, and perfect datasets are available modelling the lithospheric field at high resolutions, say at a minimum wavelength of $5 \mathrm{~km}$ for instance, would require a maximum degree of 8000. This is equivalent to finding more than 6 million Gauss coefficients by an inverse problem. Similarly, any prediction over a local area would require the same gigantic number of parameters. The significance of this latter problem will increase as more accurate data become available and it represents a strong incentive to find new representation techniques.

The prime motivation of this paper is to find an alternative to conventional global modelling and to by-pass some of these drawbacks. A regional modelling philosophy is applied in the present study, and the Revised Spherical Cap Harmonic Analysis (R-SCHA, Thébault et al., 2006) is used in order to process CHAMP data. Provided a good distribution and a sufficiently large data set, R-SCHA offers good continuity between adjacent patches and offers a possibility 


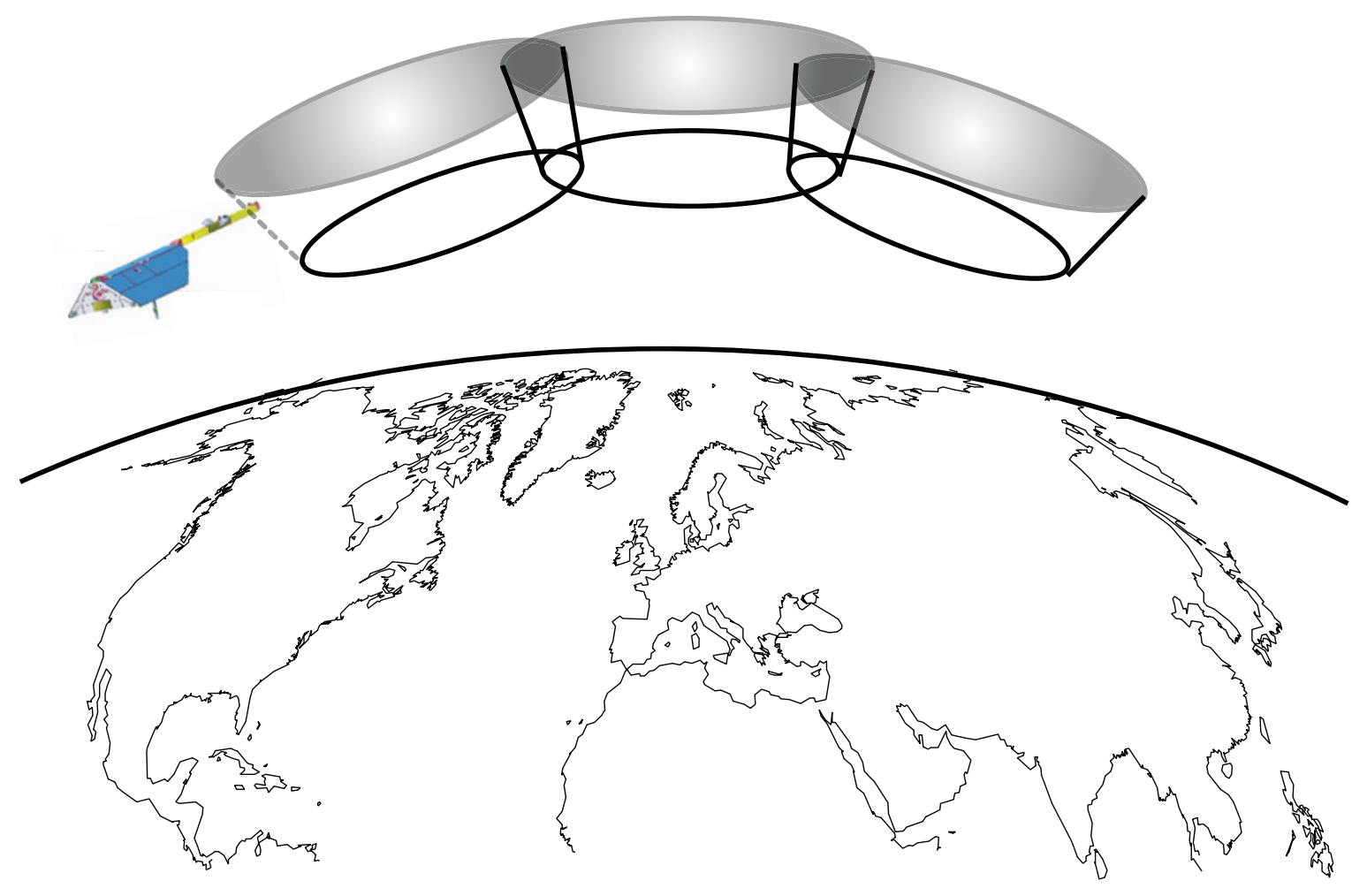

Fig. 1. Between $350 \mathrm{~km}$ and $480 \mathrm{~km}$ of altitude, the Earth is covered by overlapping cones.

to obtain the first global view of the magnetic field using a piecewise modelling procedure. Compared to spherical harmonics, solving the problem by successive regional models presents several advantages. R-SCHA is purposefully designed to accurately represent weak and small-scale fields over a given region with fewer parameters, so it is convenient for investigating remaining un-modelled lithospheric signals contained in CHAMP data. Furthermore, R-SCHA combined with the least-squares procedure assumes Gaussian noise inside each patch, not at a global scale. Thus, local noise will have no influence on adjacent areas when solving the inverse problem by regular least-squares. Therefore, local models can be individually examined and unreliable local models can be easily removed, reworked and replaced with better versions. The same flexibility would be allowed if some regions, like the polar and equatorial regions, required independent analysis and constraint.

The outline of this paper is as follows. In a first part, a brief summary of R-SCHA modelling and its use for the representation of the lithospheric field over the globe at satellite altitude is provided. This is followed by a discussion concerning the magnetic anomalies observed in the new global view, some of them being clearly visible for the first time. After a section reviewing the different techniques applied for converting regional models into a global one, features of main interest are reported and comparisons with existing lithospheric models are provided.

\section{Basic Principles}

The Revised Spherical Cap Harmonic Analysis (RSCHA) is a revision of the Spherical Cap Harmonics (SCHA) pioneered by Haines (Haines, 1985). In a source free region, the Laplace equation is solved inside a cone, and boundary conditions are applied to each surface:

$$
\begin{aligned}
\Delta V & =0 \\
\left.V\right|_{\partial_{\theta_{0}} \Omega} & =F(r, \varphi) \\
\left.\frac{\partial V}{\partial r}\right|_{\partial_{a} \Omega} & =-G_{a}(\theta, \varphi) \\
\left.\frac{\partial V}{\partial r}\right|_{\partial_{b} \Omega} & =G_{b}(\theta, \varphi)
\end{aligned}
$$

where $\partial_{\theta_{0}} \Omega$ is the lateral surface defined by the semiaperture $\theta_{0}$. The lower and the upper surfaces of radius $a$ and $b$ respectively, are defined by $\partial_{a} \Omega$ and $\partial_{b} \Omega$ (Fig. 1). This boundary value problem is split up into two parts and each sub-problem is solved separately. Such a boundary value problem provides a complete set of basis functions, and it is shown in Thébault et al. (2003) that it is the most appropriate for the study of the magnetic field. The potential can be written in terms of three infinite series expansions (Thébault et al., 2006):

$$
\begin{aligned}
V(r, \theta, \varphi)= & a \sum_{k \geq 1} \sum_{m \geq 0}\left(\frac{a}{r}\right)^{n_{k}+1} \\
& \times\left(G_{n_{k}}^{i, m} \cos (m \varphi)+H_{n_{k}}^{i, m} \sin (m \varphi)\right) P_{n_{k}}^{m}(\theta) \\
+ & a \sum_{l \geq 1} \sum_{m \geq 0}\left(\frac{r}{a}\right)^{n_{l}} \\
& \times\left(G_{n_{l}}^{e, m} \cos (m \varphi)+H_{n_{l}}^{e, m} \sin (m \varphi)\right) P_{n_{l}}^{m}(\theta) \\
+ & a \sum_{p \geq 0} \sum_{m \geq 0} R_{p}(r) \\
& \times\left(G_{p}^{m} \cos (m \varphi)+H_{p}^{m} \sin (m \varphi)\right) K_{p}^{m}(\theta)
\end{aligned}
$$


with the integer orders $m$ but non-integer degrees $n_{k}$ and $n_{l}$. The functions $P_{n_{k}}^{m}$ and $P_{n_{l}}^{m}$ are the associated Schmidtnormalised Legendre functions, while $K_{p}^{m}(\theta)$ are the conical, or Mehler, functions (Thébault et al., 2006). The constant $a$ represents the mean Earth's radius. For each cone covering the Earth, the best set of parameters $\left\{G_{n_{k}}^{i, m}, H_{n_{k}}^{i, m}\right\}$, $\left\{G_{n_{k}}^{e, m}, H_{n_{k}}^{e, m}\right\}$, and $\left\{G_{p}^{m}, H_{p}^{m}\right\}$ fitting the CHAMP data are determined by least-squares. Linear relationships between R-SCHA parameters and spherical harmonics Gauss coefficients can also be derived and used to add a priori information that constraint local models. Henceforth, the terminology 'local' or 'regional' models will refer to results obtained using R-SCHA or arrangements of R-SCHA models, in contrast to the terminology 'global' that refers to models derived in spherical harmonics.

R-SCHA is used to iteratively model the CHAMP satellite data between altitudes $a=350 \mathrm{~km}$ and $b=480 \mathrm{~km}$. The semi-apertures of all cones are set to $\theta_{0}=7^{\circ}$. Each cone is centered on a node of a quasi-regular grid described below (see Section 5). The angular distance $d$ between two nodes is $d=9.4^{\circ}$, therefore the maximum overlap between two adjacent cones is approximately $5^{\circ}$ (Fig. 1). 560 cones are needed to completely cover the Earth. The minimum wavelength represented is $\lambda \simeq 60 \mathrm{~km}$ and is equivalent to a spherical harmonics expansion to degree $n=710$. Given the resolution of CHAMP data, such a series expansion may appear unnecessary, but it provides a better fit to the data, reduces ringing effects due to truncation of the local series expansions and helps localize magnetic anomalies via better definition of their contours.

\section{Data Selection and Processing}

There are two different philosophies in global modelling. Either the magnetic field is represented using comprehensive basis functions (Sabaka et al., 2004) or it is represented after filtering and data selection (Maus et al., 2006). In the comprehensive approach, the different contributions of the magnetic field are jointly inverted using appropriate basis functions. Thus, the overlap and the contamination from a magnetic field contribution into another is considerably reduced. The main disadvantage is that, since many parameters must be found at once, the conditioning of the inverse problem degrades at relatively low harmonic degrees. Moreover, day-side data must be incorporated in the modelling, so the maximum degree describing the lithospheric field is thus limited to 65 . The second technique is based on more subjective criteria and data processing: each nonlithospheric contribution of the magnetic field is, to some extent, identified and filtered out.

The present version of R-SCHA does not allow the magnetic field representation in a comprehensive way. In particular, there is an ambiguity concerning internal and external field separation (Thébault et al., 2006). As a result, the simplest way to proceed is to rely on data selection and processing.

\subsection{Data selection}

Four years of CHAMP scalar and vector data from August 2000 to July 2004 were used in the present study. The data were selected using the same procedure as for MF3 (Maus et al., 2006 for the data selection procedure) but with a larger time span. Data were selected for quiet periods with $K_{p} \leq 2$ with a maximum of $K_{p}=2.5$ for the previous three hours from the local time sector 0:00 to 5:00 LT. The maximum $\left|D_{s t}\right|$ index chosen was $50 \mathrm{nT}$ and $|d D s t / d t|=3$ nT per hour. Filters were also applied to correct the star camera misalignment. A preliminary external field was removed from the data as well as external quadrupoles. Mid latitude data were corrected for the polar electrojet (Maus et al., 2006) and for an ocean tidal model Tyler et al. (2003) and at last, an internal core field model up to $n=15$ was removed using POMME 2.5 model (Maus et al., 2004) so that only lithospheric field, and part of the noise, remained. A number of 5359523 scalar data and 1731810 vector data locations were considered, giving an overall of 10554953 data near-homogeneously distributed over the sphere. It is worth mentioning that, unlike the MF lithospheric family of models, vector data are considered at all latitudes in the present study. Indeed, the modelling being carried out regionally, inclusion of vector data helps to better constraint the local models at high latitudes.

\subsection{Modelling the lithospheric field}

For each of the 560 cones, the data inside are rotated into the cone's reference frame. The least-squares solutions are estimated using the local basis functions. When vector and scalar data are analyzed simultaneously, the inverse problem is not linear. However, since the crustal field is quasi-static and weak, the problem can be linearized by approximating the intensity anomaly $\delta F$ at satellite altitude with:

$$
\delta F \simeq \frac{\delta \mathbf{B} \bullet \mathbf{B}_{\text {main }}}{\left\|\mathbf{B}_{\text {main }}\right\|}
$$

The intensity anomaly field is assumed here to be the projection of the lithospheric magnetic field $\delta B$ in the direction of a core field model $B_{\text {main }}$. The main field used here is the POMME 2.5 model (Maus et al., 2004) as it is the core field removed from the data. Because of good data coverage, local damping was necessary only in the polar caps where little data is available around the geographic poles (due to orbital dynamics of CHAMP). A priori information given by the relationships between global MF3 Gauss coefficients and local estimated parameters (Thébault et al., 2006) is also used. As a result, the lithospheric model in a cap of about $5^{\circ}$ near the geographic poles is very similar to MF3. In each cone, a number of 946 parameters was determined. Altogether, for the entire Earth, 529760 local parameters were found. In comparison such a resolution would require, for a maximum degree $n=710,505520$ Gauss coefficients in spherical harmonics.

There is no rigorous formulation giving the overall number of local parameters necessary to represent the field everywhere on a sphere (M. Holschneider, personal communication). Two different cases may be envisaged. On one hand, if we consider a local model as the restriction of a global one that must be zero outside the domain, the required number of local parameters is infinite, even for a single patch. On the other hand, if we discard the behavior of the model outside the domain of interest, a good estimate inside a single patch can be found with less parameters than in spherical harmonics. In practice, when stitching together 


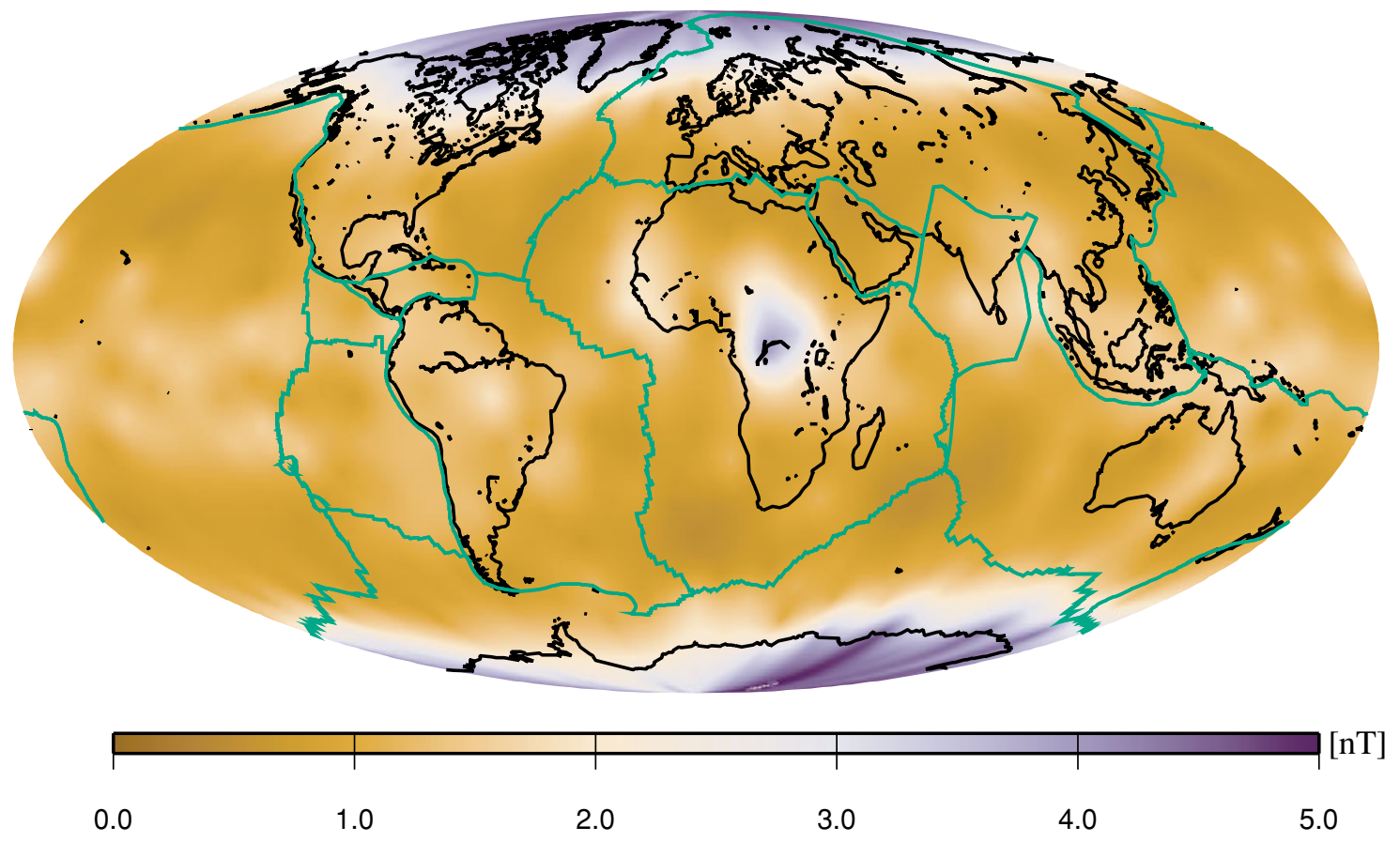

Fig. 2. Standard deviation estimated inside each cone represented on the Sphere (in nT).

local models the main interest is the magnetic field continuity between adjacent areas. Therefore, the overall number of parameters is usually greater than the number of Gauss coefficients but a good trade-off between the number of parameters and the continuity can be easily found, as it is the case here.

The standard deviation, $\sigma$, estimated inside each cone provides information about the misfit distribution at the global scale. In Fig. 2 very clear contours defining polar regions are noticeable and the misfit in these regions may reach $5 \mathrm{nT}$. At mid and low latitudes, the misfit is generally about 0.5-1 nT except in Africa, over the Bangui region. This is a good illustration of the advantage of merging together regional models. Statistically, there is no explanation for the noise increase and this artifact is generated by a pure lack of fit. Taking out the corresponding cone and expanding the local series to larger degrees reduces the misfit to the expected level and provides a model more consistent with the adjacent areas. Indeed, Bangui and Kursk anomalies, have a signature of $22 \mathrm{nT}$, making them the strongest anomalies at $400 \mathrm{~km}$.

Although not prominent, a slight increase of the standard deviation surrounds the magnetic equator and is certainly induced by the equatorial electrojet (Fig. 2).

\section{The Model at $400 \mathrm{~km}$ of Altitude}

Having found the proper set of parameters, the forward problem is computed for each cone at an altitude of 400 $\mathrm{km}$. Since the orbit of CHAMP is slightly elliptical, this altitude is common to every cone and is approximately the mean altitude of the entire dataset. This is equivalent to performing an upward/downward continuation inside each cone in order to have a regular distribution of points at a constant altitude.

Even if the Laplace equation is verified inside each cone, the divergence-free condition may not be satisfied at a global scale because cone edges produce artificial sources. This is the major risk when patching together regional models. It is therefore important to have a good overlap between adjacent cones, or to expand the local series to high degrees. In order to reduce this effect, a thin ring of $0.1^{\circ}$ was systematically removed near the cones boundaries and this precaution guarantees a better model continuity between neighboring cones. A divergence operator was applied on the synthetic data in order to check that no footprint or circular spurious anomalies are identified after removing the edges and merging the local models.

Figure 3 shows the vertical component of the magnetic field. The anomalies, have typical amplitudes between \pm 22 $\mathrm{nT}$ and are greater than what is represented using spherical harmonics. Another point of interest is the visible longitudinal oscillations. Usually, when dealing with bounded domain, a ringing arises when the local series expansion are not expanded to a sufficient truncature index. However, this is not the case here, as this possibility was investigated and eliminated. There are several methods for modifying the coefficients in a truncated series in order to reduce Gibbs effects. In particular, an attractive feature of arithmetic means in series expansions is that Gibbs phenomenon and ringing do not occur Walker (1988, chapter 2). As a result, a possible way to avoid ringing effects is to apply the generalization of the Fejér partial sum theorem (Fejér, 1911) which states that if an infinite series converges to the sum $s$, it can be approximated by its arithmetic mean expansion that converges to the same $s$ (Walker, 1988, chapter 2, section 7.2). In this method, if $K$ harmonics are included in the truncated series expansion, then the amplitude of the $n$th harmonic is weighted by $(1-n / K)$. Therefore, the amplitudes of the highest frequencies are damped towards zero. When applied to each of the series indices $k, l$ or 

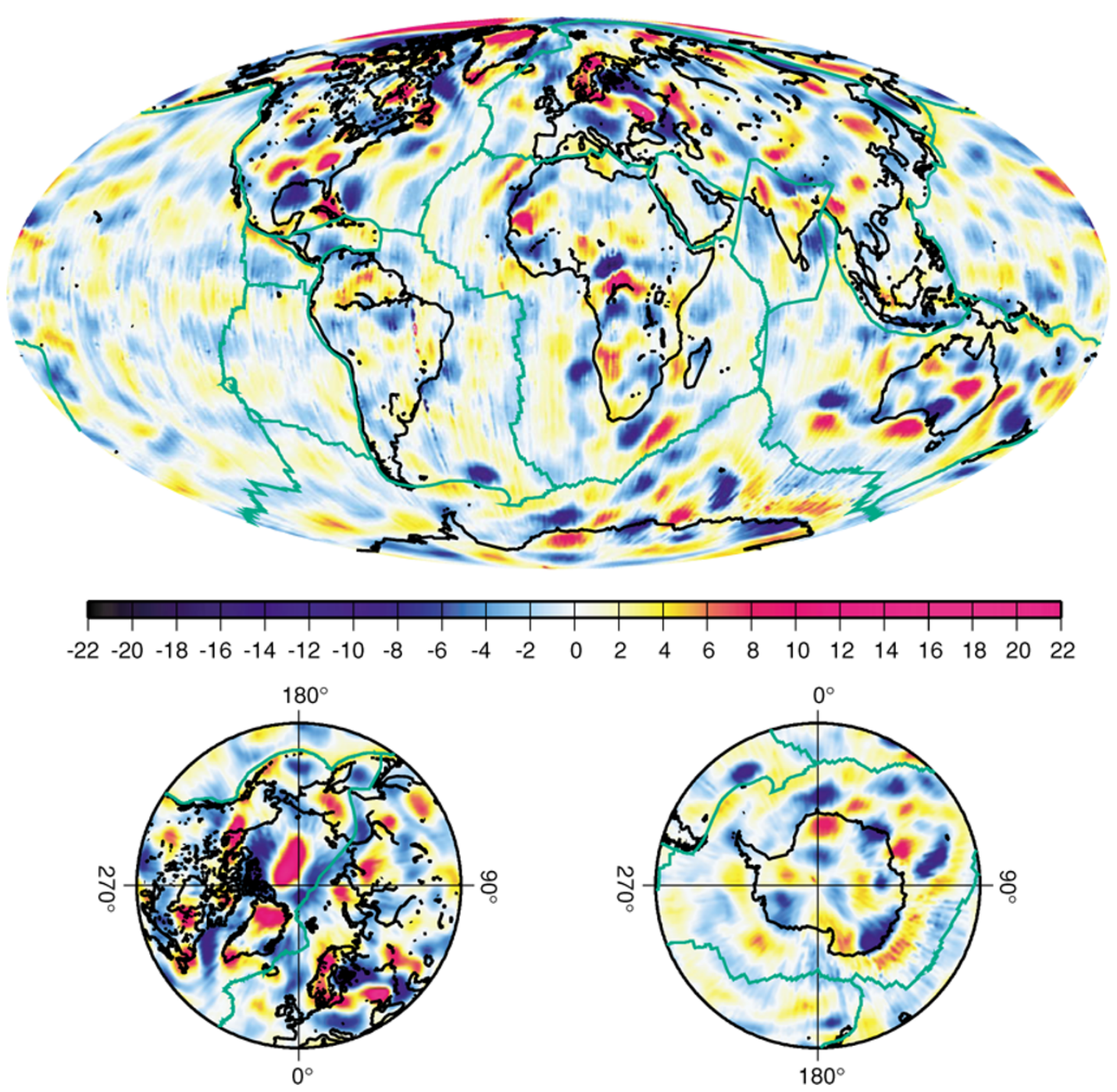

Fig. 3. Z component of the crustal field at $400 \mathrm{~km}$ modelled by R-SCHA basis functions.

$p$ in Eq. (2), this produces a great improvement in convergence properties of the local harmonics. The predictions using Fejér series and the normal series expansion showed the same longitudinal stripes, which proves that this signal is not an artifact. The line-levelling and satellite attitude errors are likely to produce such signal, and it is suspected to be the primary source of error in the dataset. This artifact should not be confused with oceanic stripes that were not clearly detected here. Considering the maximum resolution used herein and the capabilities of Swarm mission, the present procedure will certainly help to accurately delineate the oceanic stripes in the future.

The general anomaly features shown in Fig. 3 are in good agreement with what is seen using the MF3 or CM4 lithospheric models. The large-scale structures agree well, but details show many striking differences. The purpose here is not to discuss the related geology of these anomalies but a few prominent differences between the local and global representations are worthy of discussion.

The known major anomalies generally are sharper. Weaker and smaller anomalies are also better defined. The Kursk anomaly now appears as an independent unit and is no more related with the Tornquist-Tesseyre Zone. Small scale anomalies over continents, like the anomalies associated with South American craton or the African craton, are distinguished. In the Mediterranean sea there is a strong anomaly, possibly associated with active subduction under the Italian island of Sicily. Between Mozambique and Madagascar lies a strong positive anomaly in the ocean. This interesting anomaly, apparently produced by oceanic crust, can not yet be explained by simple geology (Hemant, 2003). Anomalies that are only guessed in global models are now well represented. In north-western Saudi Arabia, for example, a strong localized anomaly is observed. It is sometimes difficult to discriminate between signal and noise in local modelling, especially if they are of the same level; R-SCHA being liable to contain part of the noise. Nevertheless, although it is shown below that the model over this region is altered by noise, this previously less prominent anomaly is well constrained by the data and is thought to be genuine. Unfortunately freely distributed aeromagnetic data could not be found over this area, and, since this region is covered by sand, geological maps are unable to explain the anomaly. It nevertheless lies north of the known geological boundary of the Arabian shield of Precambrian age (Goodwin, 1991). Other regions showing 
small spots of similar nature are likely to be genuine, but a thorough investigation is required to verify these anomalies.

\section{From Local to Global Analysis}

The downward continuation of the R-SCHA local models to the Earth's mean radius proved to be hazardous. In order to check the quality and the consistency of the local modelling patches, a lithospheric model that can be compared to known spherical harmonic models, either visually or by spectral analysis, is needed. It is therefore important to estimate the new model, between ground and satellite altitudes, in terms of a spherical harmonic set of parameters. Because the CHAMP data were accurately modelled in each cone, the synthetic data built with R-SCHA now have less noise. A better "signal to noise" ratio is expected and using the local models as an input for direct integration should allow the recovery of previously discussed small scale features.

\subsection{Integration on the sphere}

Finding analytical relationships recombining all the local parameters into a proper set of unique Gauss coefficients represents the ideal situation. Unfortunately, if such relationships exist between global and local modelling, the reverse is untrue (Thébault et al., 2006). In order to by-pass this drawback, several techniques based on numerical integration can be employed for converting a set of local models to a global model.

The radial component of the magnetic field, expanded in terms of local models around the Earth at a given radius $r$, may be defined by $\widetilde{f}(r, \theta, \varphi)=-\frac{\partial V}{\partial r}$ with $V$ defined by Eq. (2). The same magnetic field component may be expanded in spherical harmonics so that:

$$
\begin{aligned}
f(r, \theta, \varphi)= & \sum_{n=1}^{N} \sum_{m=0}^{n}(l+1)\left(\frac{a}{r}\right)^{n+2} \\
& \times\left\{g_{n}^{m} \cos (m \varphi)+h_{n}^{m} \sin (m \varphi)\right\} P_{n}^{m}(\cos \theta)
\end{aligned}
$$

with $P_{n}^{m}(\cos \theta)$ representing the Schmidt-normalised associated Legendre functions, $\left\{g_{n}^{m}, h_{n}^{m}\right\}$ the set of Gauss coefficients, and $a$ the mean Earth's radius, which is generally set to $a=6371.2 \mathrm{~km}$. The equivalence of both representations allows $f(r, \theta, \varphi)=\widetilde{f}(r, \theta, \varphi)$ everywhere and, using the orthogonality properties of spherical harmonics, it yields:

$$
\begin{aligned}
\left\{\begin{array}{l}
g_{n}^{m} \\
h_{n}^{m}
\end{array}\right\}= & \frac{2 l+1}{4 \pi} \int_{0}^{2 \pi} \int_{0}^{\pi} \tilde{f}(r, \theta, \varphi)\left(\frac{r}{a}\right)^{n+2} \\
& \times\left\{\begin{array}{c}
\cos (m \varphi) \\
\sin (m \varphi)
\end{array}\right\} P_{n}^{m}(\cos \theta) \sin \theta d \theta d \varphi
\end{aligned}
$$

In practice, the Gauss coefficients defined by (5) can now be estimated. Knowing the functions $\widetilde{f}(r, \theta, \varphi)$ everywhere, thanks to the 560 sets of local parameters and the local functions (Eq. 2), the most natural choice to evaluate the coefficients, is to perform a double integration on the sphere. Without caution, the double integration is unstable (Press et al., 1992) and, because of the number of local models, is too demanding in terms of computer resources. An elegant way of by-passing this problem is to split the integration in latitudes and longitudes and compute the Legendre functions on the Legendre knots (Cain et al., 1989). The problem may be thereby preconditioned by computing discrete values of the function $\widetilde{f}(r, \theta, \varphi)$ on a particular grid adapted for the Gauss-Legendre quadrature. The grid can also be created using new sampling theorems requiring more points on the sphere but involving less numerical operations for the evaluation of the spherical transform Driscoll and Healy (1994). Such an algorithm was adapted and improved by Lesur and Gubbins (1999) and more details about the integration techniques can be found in references therein.

Here, another solution was adopted, and these powerful and elaborated methods were not implemented. Although it requires at least twice as many points, a simple alternative consists in computing the synthetic data on a regular grid and finding the Gauss coefficients individually by leastsquares. The principle is to use the discrete formulation of (5) and to build a grid having regular surface elements. If the grid is irregular, the discretization of (5) is not accurate and Gauss coefficients have to be simultaneously found by a unique inverse problem and thus, high degrees may be aliased.

Defining a regular grid on a sphere has been a long standing problem in many branches of science. Approximations of regular grids are usually given by the projection of a polyhedron over the sphere (Dufour, 1991). For the present purpose these geometric grids are not suitable. Since the maximum difference between two surface elements varies from $1 \%$ to $10 \%$, the Gauss coefficients can be reasonably estimated by (5) only up to degree 90 or degree 34 respectively. Using a different philosophy, Saff and Kuijlaars (1997) created a suitable grid by considering the equilibrium of electrically repellent particles on a sphere. For the present study, a mesh of 263850 points was created on the sphere using the method of Saff and Kuijlaars (1997). The grid proved to be regular within $0.01 \%$ of uncertainties and, as a result, Gauss coefficients could be assumed to be independent and could be estimated individually by least-squares at least up to degree 180 . Since the maximum spherical harmonic degree expected to be represented by Swarm data (ESA report, 2004) is 150, this simple method should apply efficiently.

\subsection{Residual analysis}

If local and global modelling were both equivalent in the finite approximation, their difference would converge to zero. However, Eq. (5) is an equality in the mean square sense and does not necessarily imply that the local model converges simply towards the global one. Therefore, localized differences are expected when looking at the residual maps. Figure 4 shows the residual distribution that cannot be represented by spherical harmonic internal basis functions. The average magnitude of the remaining signal is about $0.40 \mathrm{nT}$ and its maximum absolute value of $8 \mathrm{nT}$ lies near Antarctica. The residual over the sphere has three kinds of organized features.

Most small-scale residuals seem to be lithospheric continental anomalies of small spatial resolution. The spherical harmonic model is therefore necessarily smoother than the view showed on Fig. 3. North of the Arabian shield, as well as over the Bangui anomaly lithospheric fields are still identified in the residuals. Since local models require 

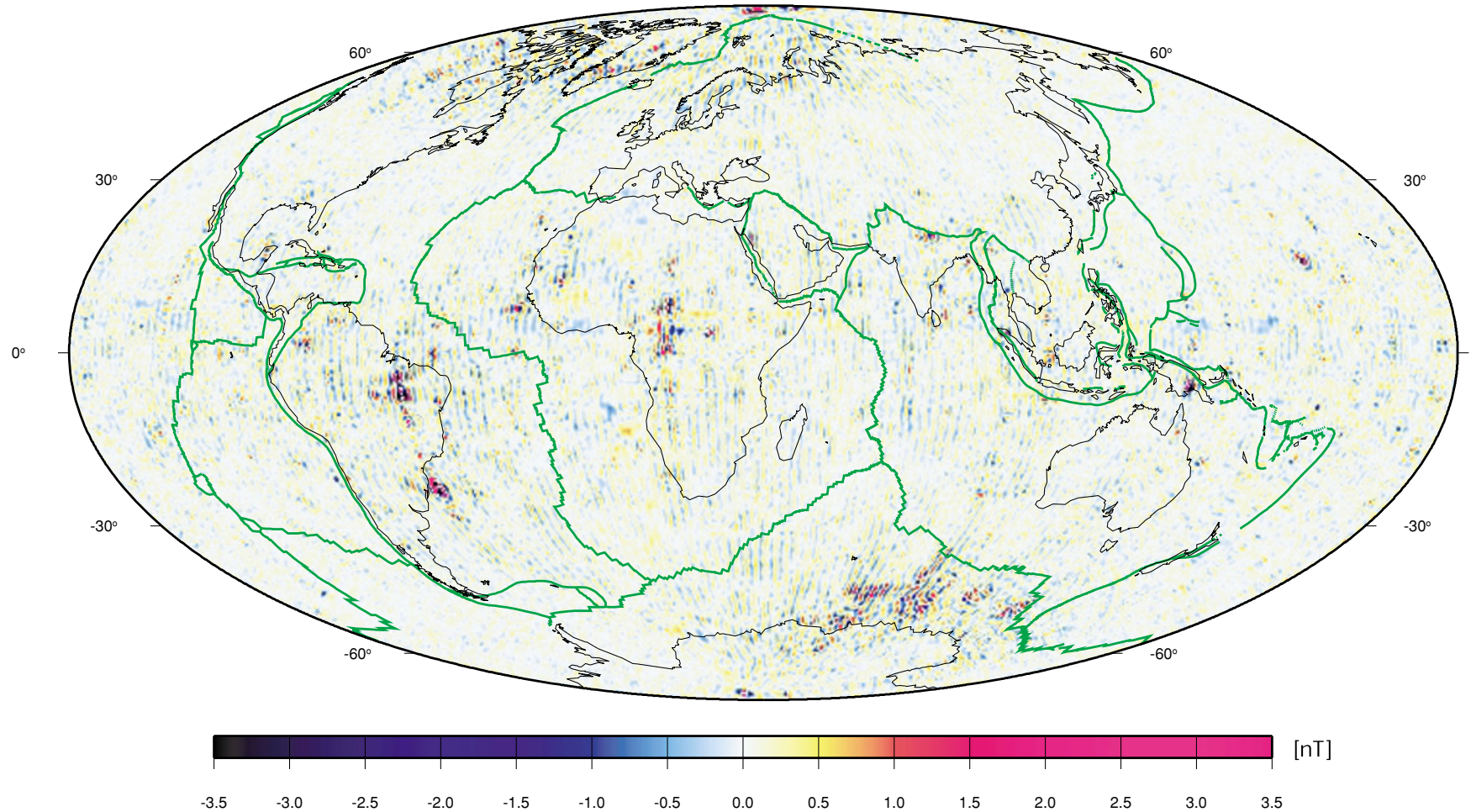

Fig. 4. Remaining signal after transformation of the crustal field depicted in Fig. 3 into Spherical Harmonic model of maximum degree $n=150$.

larger series expansion over that latter regions, it is likely that high spherical harmonics are also needed to represent them accurately. A strong signal is visible in the northern part of the Brazilian shield and apparently belongs to a large noisy drag starting from Venezuela and extending into northern part of Brazil. Other smaller and weaker signals can also be associated with known geological features: the South Himalayan Belt anomaly in the North of India and the anomaly in New Guinea are easily identified. Both features are coincidentally too close to subduction zones to be entirely attributed to noise. Very weak signals, where noise is probably dominant, are noticeable over some areas such as Sri Lanka or the East Canary plateaus. At last, in oceanic regions, one of the strongest residuals can be found west of the Hawaiian Islands, over the Mid-Pacific Mountains.

Isolated spikes over both oceans and continents should be regarded as pure noise since their spatial extent is generally lower than hundred kilometers. Moreover, most of the remaining signal is buckled by a north-south trend and high frequency east-west oscillations. The reason invoked for these stripes by Maus et al. (2006) concerns the lineleveling issues, which is the main source of this signal.

One remaining signal dominates at the transition between mid and high latitudes (around the geomagnetic latitudes $\pm 65^{\circ}$ ). Its ring-like ribbon indicates that it is probably a polar electrojet remaining contribution. Note that the present study incorporates both vector and scalar at all latitudes and vector components are very sensitive to the ionospheric field. Moreover, this signal follows the boundaries of the polar regions where the standard deviation increases (see Fig. 2). Another interesting assumption may be put forward: because low and high latitudes exhibit very different properties in terms of ionospheric currents, the vector and scalar datasets are processed differently within these two regions Maus et al. (2006). The overlapping zone between the two methods of processing lies around the geomagnetic latitudes $\pm 65^{\circ}$ and this may produce thin step zones that cannot be represented by spherical harmonics of low degrees only.

It is worth mentioning that there is no evidence of artificial noise created by edge effects in the local modelling, even of very low amplitude, and the stitching together of regional models has no direct impact on the final residual. If existing, this effect is a second order noise.

\subsection{Spectra and correlation}

The spectrum is an interesting tool allowing a visual comparison between different models (Lowes, 1966). In Fig. 5, the spectrum of the current model is compared with an Ørsted model (Olsen et al., 2002), CM4 (Sabaka et al., 2004), MF3 and MF4 (Maus et al., 2006) from degree 16 to 71.

At a first sight, the quality of the new model lies between the CM4 and MF4 models (deep-blue line in Fig. 5). It is more stable than the CM4 above $n=36$, but since the CM4 also incorporates day-side data, this improvement may be exclusively due to data selection. The obtained model starts degrading above $n=56$, and spectral contributions higher than $n=65$ are not acceptable as they are inconsistent at the mean Earth radius. This may appear in contradiction with results presented in Fig. 3 where small wavelengths were clearly defined but in fact, since the spectrum is computed at the reference surface, the downward continuation is very sensitive to the noise in the data. The quasi-periodic high east-west oscillations are probably the main effect hampering the spherical harmonics representation (Fig. 4). Indeed, they dominate the small-scale structures and have apparently a global structure while small scale signal are 


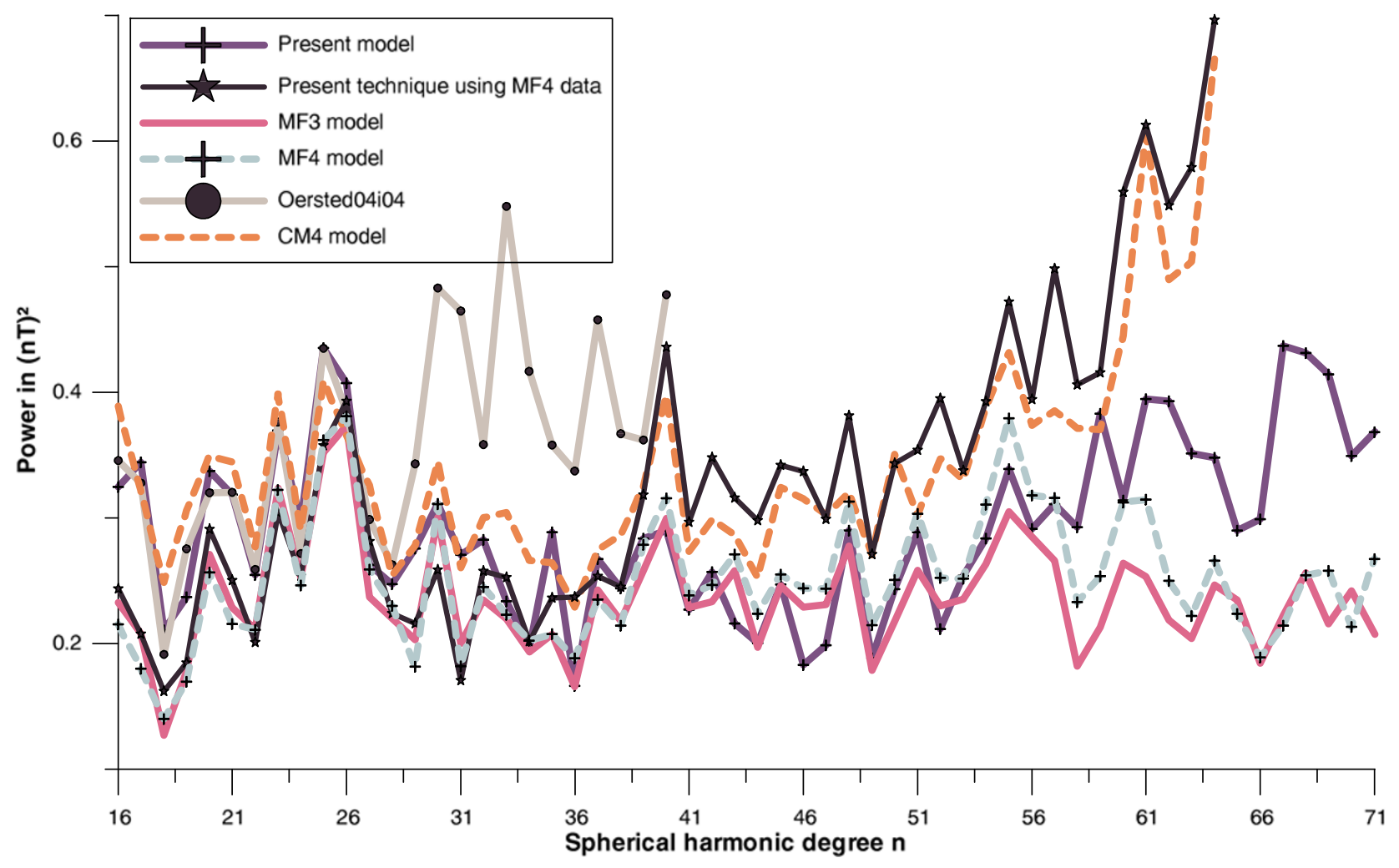

Fig. 5. Normalized Mauersberger-Lowe spectra (divided by $2 n+1$ ) at the mean Earth's radius for several SH models.

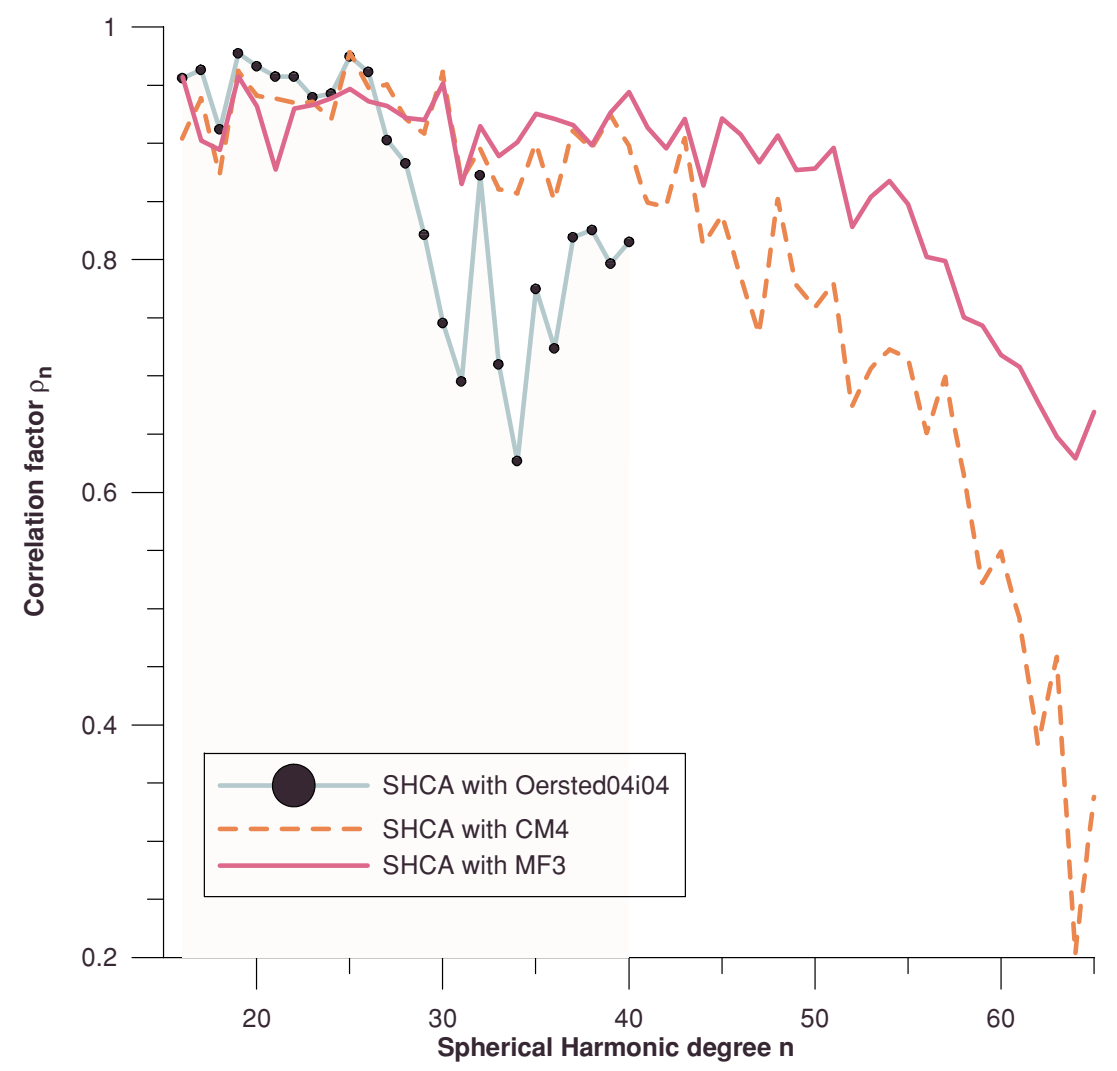

Fig. 6. Correlation analysis between the present model and other crustal models. 

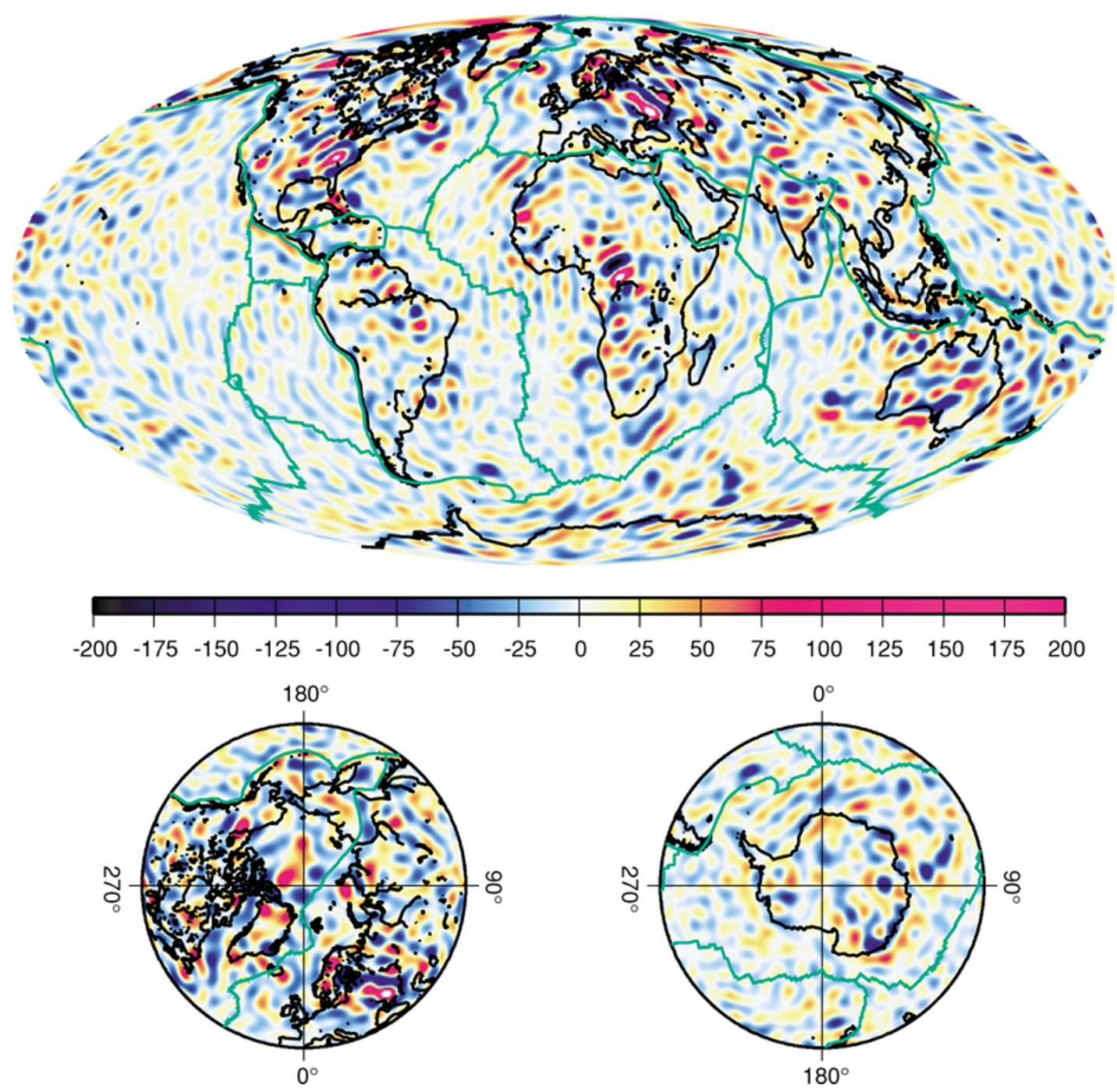

Fig. 7. Z component of the crustal model represented in spherical harmonics up to degree 65 at the Earth's mean radius.

more localized. Therefore, line-leveling noise affects relatively low spherical harmonic degrees. Moreover, as already said, some external field noise is represented by the R-SCHA modelling. For these reasons, it remains difficult to estimate a lithospheric spherical harmonic model without regularization, as it is the case here.

The behavior of the new model between degrees $n=16$ and 32 has an intriguing characteristic. Although the same data correction as for the MF3 lithospheric model was applied, it shows a better agreement with the Ørsted and CM4 models than with the MF model family. A spherical harmonic correlation analysis (Langel and Hinze, 1998, page 81) shown in Fig. 6 confirms that the model follows the Ørsted model spectrum up to degree $n=26$, then from $n=27$ to $n=31$ it is in better agreement with CM4, and then only for higher $n$ it is better correlated with MF3. The fact that MF3 is damped from degree 60 could be a reason since all Gauss coefficients, including the low degrees, will adjust to minimize the residual quadratic form.

For further comparison, the exact MF4 dataset was considered and the stitching procedure was carried out again. The selection criteria between MF3 and MF4 remain essen- tially the same although MF4 data are better corrected for polar electrojets, vector data have an improved calibration, and data disturbed by F-Region currents are better eliminated (Maus et al., 2006). This has, apparently, little effect on the large wavelengths representation in MF3 and MF4 models (Fig. 5). Therefore, the major difference between the two experiments carried out in the present study is that vector data are discarded above geomagnetic latitudes $\pm 65^{\circ}$ when using the exact MF4 data set. The new spectrum is shown in Fig. 5 and illustrates how the new model now correlates better with MF3 and MF4 from degrees 16 to 33 . It is legitimate to wonder if selecting and withdrawing data according to spatial criteria, as it is done in MF4 above geomagnetic latitude $\pm 65^{\circ}$, does not introduce a spatial bias in the modelling. This could explain the two different results observed in the first part of the spectrum when considering vector and scalar data or scalar data only in the polar regions. Unfortunately, using scalar data only near the polar caps does not guarantee a very good vector continuity between neighboring models using R-SCHA. This is why the model is unstable from degree 40. To which extent these discontinuities have an effect on low harmonics remains an 
open question. It is thereby difficult to conclude, using this procedure, if MF4 has a loss of power or, rather if CM4, Ørsted, and the present model overestimate the actual power spectrum at low degrees due to the noise in the vector data near polar caps.

Figure 7 shows the magnetic field at the reference surface using a maximum degree of 65 . The contribution from degrees higher than 65 were discarded because prominent stripes appear at the reference surface in oceanic regions and at the ocean-continent boundary near Antarctica. The major known anomalies are well represented everywhere, and in this respect, the model is robust. The Java trench, for instance, is reasonably well defined. As expected there is overall more power than MF3, and the anomalies are more prominent although noise is noticeable. One of the major results is that part of the lithospheric signal depicted in Fig. 3 is successfully represented, although it remains challenging to unravel the small-scale lithospheric signal when transforming the local set of models into a unique spherical harmonic model. In particular, the Arabian Shield anomaly is well defined. The Sicily island anomaly and the South of Turkey anomaly, lying at the junction of the African, Arabian and Eurasia plates, are also correctly represented.

\section{Conclusion}

The data recorded by satellites have different quality around the Earth and forthcoming missions will supply the scientific community with a large amount of high quality and resolution data. A primary interest is to prepare a good alternative to spherical harmonics in order to represent these data with regional constraints. In this paper, a piecewise modelling technique at high resolution based on the Revised Spherical Cap Harmonic Analysis regional modelling technique (R-SCHA, Thébault et al., 2006) is successfully applied.

Homogeneous distribution of CHAMP data is the keystone for stitching together local models. Since local series expansion can be expanded to high degrees, the continuity between adjacent models is preserved and no artifacts are noticed when merging the local models. Indeed, both the large and the short wavelengths of the lithospheric field are detected. In general, lithospheric anomalies represented by local models are sharper and better defined than what is represented with spherical harmonics. Moreover, local models clearly exhibit evidence for genuine lithospheric signal at short wavelengths. In Saudi Arabia, for instance, CHAMP detects an anomaly that is thought to originate from a real geological feature. Nevertheless, since regional models may be still contaminated by noise, some very small features are less realistic. In spite of this inherent problem, in many places the local model shows weak but evident signatures of known geological units.

In order to compare this new global view with known lithospheric spherical harmonic models, a successful transformation from local to global modelling is performed, and a set of Gauss coefficients is found. Although it has a lower resolution than MF3 and MF4 (Maus et al., 2006), the present lithospheric model is realistic. Part of the smaller anomalies seen by local models are successfully converted into a global model. Nevertheless, noise and artifacts still alter the signal and degrade the determination of the Gauss coefficients to high degrees. Therefore, stable models at the reference surface for degrees higher than 56 are difficult to obtain without regularization. The next strategy will be to develop more sophisticated methods to increase the stability of the Gauss coefficients. A simple idea to implement this is to apply a damping in the least-squares inversion (Maus et al., 2006). Another possibility is to apply particular weights to each of the regional models according to their location on the sphere. Such improvements about the methodology could help to derive a new lithospheric reference field, more stable than global models. In the framework of the World Digital Magnetic Anomaly Map (WDMAM), such a reference field is needed in order to constrain and to restore large wavelengths contained in aeromagnetic compilations. Considering the static nature of the lithospheric field, correction could also be applied retroactively to previous satellite data (Maus et al., 2006). In addition, special efforts to improve the selection of data will allow further progress in eliminating noise in CHAMP data. In this paper, for instance, the line-leveling problem has been the most difficult issue. Considering the better signal-to-noise ratio expected by the measurements of the Swarm satellites data, such a limitation will be, to some extent, less important.

A main point of this paper is that a regional modelling proved to be efficient at a global scale, although it is not primarily designed for this purpose. The ultimate goal of R-SCHA is to combine ground data with satellite data, although it may be adapted to detect any sort of weak signal. The increased resolution of the Swarm satellites will allow, for the first time, to fill the spectral hole between spherical harmonic degrees 90 and 150. A method like RSCHA is likely to succeed in combining these high resolution data with aeromagnetic data in order to provide our first ever regional models of the lithospheric fields at scales from $10-3000 \mathrm{~km}$ with a manageable number of parameters.

Acknowledgments. I would like to record my gratitude to S. Maus who processed the CHAMP datasets used in this paper. M. Holschneider and V. Lesur are thanked for their motivating and helpful discussions about regional modelling. The paper was considerably improved by the referees V. Lesur and T. Ishihara. Figures were produced using GMT (Wessel and Smith, 1991).

\section{References}

Cain, J. C., Z. Wang, C. Kluth, and D. R. Schmitz, Derivation of a geomagnetic model to $n=63$, Geophys. J. Int., 97, 431-441, 1989.

Driscoll, J. R. and D. M. Healy, Computing fourier transforms and convolution on the 2-sphere, Adv. Appl. Maths., 15, 202-250, 1994.

Dufour, H. M., A proposal for a unique quasi-regular grid on a sphere, Manuscripta Geodatica, 16, 267-273, 1991.

ESA, Swarm - the Earth's magnetic field and environment explorers, Tech Report, SP-1279(6), European Spatial Agency, http://www.esa.int/ esaLP/LPswarm.html, 2004.

Fejér, L., Sur les singularités de la série de fourier des fonctions continues, Annales Scientifiques de l'E.N.S., 28, 63-104, 1911.

Goodwin, A. M., Precambrian Geology: The Dynamic Evolution of the Continental Crust, Academic Press, San Diego, 1991.

Haines, G. V., Spherical cap harmonic analysis, J. Geophys. Res., 90, 2583-2591, 1985.

Hemant, K., Modeling and interpretation of global lithospheric magnetic anomalies, PhD thesis, Freien Universität, Berlin, 2003.

Langel, R. A. and W. J. Hinze, The Magnetic Field of the Earth's Lithosphere, Cambridge University Press, 1998.

Lesur, V. and D. Gubbins, Evaluation of fast spherical transforms for 
geophysical applications, Geophys. J. Int., 139, 547-555, 1999.

Lowes, F. J., Mean-square values on sphere of spherical harmonic vector fields, J. Geophys. Res., 71, 2179, 1966.

Maus, S., H. Lühr, G. Balasis, M. Rother, and M. Mandea, Introducing POMME, the POtsdam Magnetic Model of the Earth, http://www.gfzpotsdam.de/pb2/pb23/index.html, 2004.

Maus, S., M. Rother, K. Hemant, C. Stolle, H. Lühr, A. Kuvshinov, and N. Olsen, Earth's crustal magnetic field determined to spherical harmonic degree 90 from CHAMP satellite measurements, Geophys. J. Int., 164(2), 319-330, 2006.

Menke, W., Geophysical Data Analysis: Discrete Inverse Theory, volume 45 of International Geophysics Series, Academic Press, New York, second edition, 1989.

Olsen, N., A model of the geomagnetic field and its secular variation for epoch 2000 estimated from Ørsted data, Geophys. J. Int., 149, 454-462, 2002.

Press, W. H., S. A. Teulolsky, W. T. Vetterling, and B. P. Flannery, Numerical Recipies in C, Cambridge Unviversity Press, New York, second edition, 1992.
Sabaka, T. J., N. Olsen, and M. Purucker, Extending comprehensive models of the Earth's magnetic field with Ørsted and CHAMP data, Geophys. J. Int., 159, 521-547, 2004.

Saff, E. B. and A. B. J. Kuijlaars, Distributing many points on a sphere, The Mathematical Intelligencer, 19(1), 5-11, 1997.

Thébault, E., Modélisation régionale du champ magnétique terrestre, $\mathrm{PhD}$ thesis, Université Louis Pasteur, Strasbourg, 2003.

Thébault, E., J. J. Schott, and M. Mandea, Revised spherical cap harmonic analysis (R-SCHA): validation and properties, J. Geophys. Res., 111, B01102, 2006.

Tyler, R., S. Maus, and H. Lühr, Satellite observations of magnetic fields du to ocean tidal flow, Science, 299, 239-241, 2003.

Walker, J. S., Fourier Analysis, Oxford University Press, 1988.

Wessel, P. and W. H. F. Smith, Free software help maps and display data, EOS Trans., 72, 441, 1991.

E. Thébault (e-mail: Erwan.Thebault@gfz-potsdam.de) 